\title{
REVISIÓN DE Favosites argentina Thomas, 1905, ESPECIE TIPO DE Argentinella nov. gen., CORAL TABULADO DEL DEVÓNICO INFERIOR DE ARGENTINA
}

\author{
Esperanza M. FERNÁNDEZ-MARTÍNEZ ', \\ Yves PLUSQUELLEC ${ }^{2}$ y Francis TOURNEUR ${ }^{3}$
}

\footnotetext{
' Universidad de León. Departamento de Ingeniería Minera. c/ Jesús Rubio, 2. 24071 León. Espaina. dimefm@unileon.es

2 Université de Bretagne Occidentale. UMR 6538 Domaines océaniques, Laboratoire de Paléontologie et Stratigraphie du Paléozoïque. Faculté des Sciences. 6. Avenue Le Gorgeu. 29285 Brest Cedex. Francia. Yves.Plusquellec@univ-brest.fr

3mpasse du Blanc Bou.21.5340 Faulx-les-Tombes. Bélgica. francis.tourneur@wanadoo.be
}

Fernández-Martínez, E.M., Plusquellec, Y. y Tourneur, F. 2002. Revisión de Favosites argentina Thomas, 1905 , especie tipo de Argentinella nov. gen., coral tabulado del Devónico Inferior de Argentina. [Revision of Favosites argentina Thomas, 1905, type species of Argentinella nov. gen., a Lower Devonian tabulate coral from Argentina.] Revista Española de Paleontologia, 17(1), 101-116. ISSN 0213-6937.

\begin{abstract}
Type material of Favosites argentina Thomas, 1905, from the Lochkovian of Cerro del Fuerte (Argentine Precordillera), along with various topotypical material belonging to the same collection, is decribed and figured. The study of this material serves as a basis for establishing the new genus Argentinella. This genus is characterized by branching colonies, with a marked development of the septal elements, especially pronounced in the calices which present 12 spiny septal ridges, peripheral thickening of the walls and tabulae, and a wall microstructure where microlamellae prevail but coexist with lamellae.
\end{abstract}

Keywords: Anthozoa, Tabulata, systematic, Lower Devonian, Argentina.

\section{RESUMEN}

Se describe y figura el material tipo de Favosites argentina Thomas, 1905, procedente del Lochkoviense de Cerro del Fuerte (Precordillera Argentina), así como diverso material topotípico perteneciente a la misma colección. El estudio de este material sirve de base para el establecimiento del nuevo género Argentinella. Dicho género está caracterizado por colonias ramificadas, con un fuerte desarrollo del aparato septal especialmente acusado en los cálices que están ornamentados por 12 arrugas septales espinosas; espesamientos periféricos de la pared y las tábulas y paredes con una microestructura en la que coexisten microlamelas y lamelas.

Palabras clave: Anthozoa, Tabulata, sistemática, Devónico Inferior, Argentina.

\section{INTRODUCCIÓN Y ANTECEDENTES}

Este trabajo constituye la tercera entrega de un conjunto de investigaciones realizadas por los autores sobre los corales tabulados ramificados del Devónico de Argentina y Bolivia (ver Fernández-Martínez et al., 1999a; Tourneur et al., 2000).

El presente capítulo está dedicado a la revisión y actualización sistemática de Favosites argentina Thomas, 1905. La redescripción y refiguración adecuadas del material tipo se hacen especialmente necesarias si se tiene en cuenta que las citas de esta especie han estado, y todavía están, afectadas por errores y ambiguiedades tanto de índole estratigráfica como sistemática.

Favosites argentina es una especie creada por Thomas en 1905 a partir de material recolectado por W. Bodenbender, posiblemente en las campañas de campo de 1894 (Bodenbender, 1896; Bodenbender y Kayser, 1896). Ninguno de estos autores cita la procedencia estratigráfica exacta del material recolectado, aunque Thomas (1905) indica que los ejemplares proceden de la sección llamada Cerro del Fuerte (concretamente, y de 
acuerdo con los autores anteriormente citados, de la parte occidental de este cerro), una localidad ya clásica en la que afloran materiales de edades comprendidas entre el Ordovícico y el Devónico. Thomas (op. cit.), sin duda siguiendo las indicaciones de Bodenbender, atribuye una edad silúrica a este yacimiento.

Las citas posteriores de Favosites argentina son numerosas (un resumen exhaustivo de las mismas, con indicación precisa de las localidades, puede encontrarse en Castellaro, 1966; Baldis, 1971 y, en menor medida, Baldis, 1975) pero, en el aspecto relativo a su procedencia estratigráfica, solo añaden confusión. Así, Castellaro (1966) lista varias citas de esta especie, tanto en Cerro del Fuerte como en otras localidades, atribuyendo siempre a las mismas una edad Silúrico Medio (lo que, de acuerdo con la información existente en las hojas geológicas del momento, indicaría que proceden de la Formación Los Espejos). Y Baldis (1971) hace un detallado análisis de todas las citas de dicho taxón concluyendo que, a pesar de haber sido mencionado reiteradamente en materiales silúricos, Favosites argentina no ha sido encontrado nunca en rocas de edad silúrica probada y que, por el contrario, este coral aparece con frecuencia acompañado de fauna de edad Devónico Inferior (Baldis, 1971). Asumiendo estos datos, Favosites argentina aparecería, concretamente, en materiales de la Formación Talacasto; pero el trabajo de Baldis (op. cit.) adolece de un análisis sistemático de las citas revisadas, por lo que no podemos tener la certeza de que este material corresponda realmente al taxón de Thomas.

Por otro lado, y en los últimos años, varios trabajos han indicado un error en la asignación genérica de Favosites argentina. Así, Tourneur, en un poster presentado en la Table ronde européenne «Paléontologie et Stratigraphie en Amerique Latine», celebrada en Lyon, en Julio de 1992, realiza un estudio preliminar del material tipo de esta especie y avanza que se trataría de un nuevo género de la familia Striatoporidae. Paralelamente, Contreras y Peralta (1994) asignan al género Striatopora Hall numerosos ejemplares procedentes de diversas secciones de la Formación Talacasto en la Precordillera Argentina y que, según ellos, pueden ser atribuidos a Favosites argentina. Desgraciadamente, ambas asignaciones son realizadas en resúmenes de congresos, sin que exista una publicación adecuada del trabajo sistemático realizado.

En esencia, el problema tiene su origen no sólo en imprecisiones y omisiones históricas sino en el hecho de que el material definido por Thomas como Favosites argentina guarda una gran similitud en su morfología externa con formas del género Parastriatopora que han sido halladas, sin ninguna duda, en la Formación Talacasto (Fernández-Martínez et al., 1999a). Es, por tanto, muy probable que algunas de las citas de Favosites argentina en materiales de dicha formación correspondan, en realidad, a Parastriatopora sanjuanina Fernández-Martínez, Plusquellec y Tourneur, 1999.

En este contexto, nuestro trabajo está exclusivamente dedicado a revisar el material tipo de la especie Favosites argentina sin profundizar en el estudio de formas recolectadas posteriormente y que requieren revisiones estratigráficas y sistemáticas individuales.

El material tipo de Favosites argentina está constituido por cuatro ejemplares (un lectotipo y tres paralectotipos), así como por 15 ramas halladas junto a éste y que sin duda tienen idéntica procedencia. Todo él se encuentra actualmente depositado en la colección del Instituto y Museo Geológico y Paleontológico de Göttingen (Alemania) con las siglas IMGPGö (ver apartado de Material). El estudio detallado del citado material y su comparación con formas próximas sugiere, como ya avanzó Tourneur (1992), la necesidad de crear un nuevo género para acoger a esta especie argentina.

\section{CONTEXTO GEOGRÁFICO Y ESTRATIGRÁFICO}

La Precordillera Argentina es un sector individualizado adyacente a la Región Andina, con forma de franja alargada, situada en el área centro-occidental de Argentina (ver Fig. 1 en Herrera, 1995). Está constituida por diversas rocas marinas del Paleozoico y contiene numerosas secciones ya clásicas en el estudio de los fósiles de América del Sur.

Las muestras descritas proceden de Cerro del Fuerte (flanco occidental), una sección situada unos 12 kilómetros al sureste de Jáchal (provincia de San Juan), en la parte norte de la Precordillera argentina [una figura con la ubicación precisa de este yacimiento puede encontrarse en Herrera (1995)]. Como se indicó anteriormente, se desconoce la procedencia estratigráfica exacta del material estudiado por Thomas, pero el análisis de esta sección sugiere que los ejemplares tipo podrían proceder o bien de los niveles superiores de la Formación Los Espejos, o bien de la Formación Talacasto, en cualquier caso, siempre de materiales de edad Lochkoviense.

La Formación Los Espejos es, en su mayor parte, de edad silúrica pero sus niveles superiores en la sección de Cerro del Fuerte corresponden ya al Lochkoviense (Benedetto et al., 1992). Se trata de fangolitas bioturbadas y caracterizadas por una coloración vinosa que contrasta con las pelitas verdes sobre las que se sitúan, y cuya base contiene dos o tres niveles de acumulación con fragmentos de braquiópodos, briozoos y corales (Benedetto et al., op. cit.). Es importante advertir que estos autores señalan la presencia de estos niveles lochkovienses en la localidad de Cerro del Fuerte, pero no en otros afloramientos de la Formación Los Espejos.

La otra opción de procedencia es la Formación Talacasto, la cual ha librado algunos corales tanto en Cerro del Fuerte como en otras localidades. Esta formación ha sido datada mediante braquiópodos como Lochkoviense-Emsiense inferior (Herrera, 1993; 1995). El estudio de palinomorfos recolectados en el yacimiento de Cerro del Fuerte ha confirmado esta datación (Le Herissé et al., 1997). En cualquier caso, los niveles con corales de la Formación Talacasto son de edad Lochkoviense. 
De esta formación, y concretamente de su afloramiento en la localidad de Quebrada La Cortadera o Los Algarrobos (situada en la rama sur de la Precordillera Argentina), procede el material tipo de Parastriatopora sanjuanina Fernández-Martínez, Plusquellec y Tourneur, una especie que también aparece en la localidad de El Tambolar (Fernández-Martínez, et al., 1999a).

Por tanto, resulta difícil con los datos actuales reconocer la procedencia exacta del material tipo, aunque existen dos elementos que parecen apuntar a la Formación Los Espejos. Por un lado, la pátina rojiza que muestran las ramas depositadas en Göttingen, la cual apenas se observa externamente pero cuya presencia se advierte claramente en varias láminas delgadas. Por otro lado, recientes recolecciones en la sección de Cerro del Fuerte (Carrera, com. pers.) parecen sugerir la presencia de la especie de Thomas en dichos niveles, por lo que un estudio más detallado de estas nuevas recolecciones se encuentra actualmente en curso (Carrera, Rodríguez y Fernández-Martínez, en prep.).

En cualquier caso, e independientemente del nivel preciso de recogida, puede asignarse a los ejemplares tipo una edad Lochkoviense.

\section{SISTEMÁTICA}

CLASE ANTHOZOA Ehrenberg, 1834

SUBCLASE TABULATA Milne-Edwards y Haime, 1850

ORDEN FAVOSITIDA Wedekind, 1937

Familia Parastriatoporidae Tchudinova, 1959 Género Argentinella nov. gen.

Derivatio nominis: De Argentina, país de origen de este género.

Especie tipo: Favosites argentina Thomas, 1905.

\section{Diagnosis}

Coral ramificado, con ramas cilíndricas constituidas por coralitas que desembocan perpendicularmente al exterior. Cálices profundos, con paredes verticales ornamentadas por 12 arrugas muy desarrolladas y coronadas por alineamientos de espinas o gránulos. Poros murales presentes en los cálices. Fuerte desarrollo del aparato septal, constituido por espinas, también en el interior de las ramas. En la zona axial, tábulas con frecuencia muy irregulares, a menudo incompletas, a veces verticales; en regiones periféricas, tábulas más regulares, en ocasiones engrosadas y con espinas. Poros de sección circular, murales y angulares. Engrosamiento distal de la pared, aproximadamente coincidente con el inicio de la curvatura de las coralitas. Lámina media granular rectilínea, fibroides presentes o no, esclerénquima principalmente constituido por grandes microlamelas, con lamelas en proporción variable.

Branching corallum, with cylindrical branches made up of corallites with perpendicular openings to surface. Deep calices with vertical walls presenting 12 highly developed ridges each of which has rows of spines and granulae. Wall pores in the calices. Marked development of septal elements, made up of spines, also present within the branches. Tabulae in the axial zone are frequently irregular in form, often incomplete, and sometimes vertical; in peripheral areas, tabulae are more regular in shape, occasionally thickened, and have spines. Wall and angular pores with circular outline. Distal thickening of wall, coinciding more or less with the start of the corallite curvature. Straight granular median suture, fibroids present or not, sclerenchyme mainly made up of large microlamellae, with lamellae in varying proportions.

\section{Discusión y comparaciones}

Argentinella nov. gen. muestra los rasgos fundamentales que caracterizan a los representantes de la familia Parastriatoporidae: colonias ramificadas, cálices que desembocan en ángulo perpendicular a la superficie de las ramas, espesamientos periféricos, poros angulares (P1) y murales (P2), aparato septal espinoso y microestructura principalmente microlamelar. En relación con este último rasgo hay que indicar que, aunque el esclerénquima mural de Argentinella está constituido de forma dominante por un tejido calcítico microlamelar, en el seno del mismo aparecen intercalaciones de elementos lamelares (ver apartado relativo a microestructura). La diagnosis familiar debería, por tanto, enmendarse en su aspecto microestructural para dar cabida a este tipo de microestructura microlamelar-lamelar.

Argentinella comparte varios rasgos morfológicos con diversos géneros de corales tabulados, todos ellos ramificados.

La diagnosis enmendada de Parastriatopora Sokolov puede consultarse en Lafuste et al., (1992a). Este género comparte con Argentinella tanto la naturaleza microlamelar de su esclerénquima como una morfología externa muy similar, aunque las colonias de Argentinella tienen un carácter más ramificado que los representantes de Parastriatopora. Las principales diferencias entre ambos géneros se encuentran en el fuerte desarrollo del aparato septal en Argentinella, así como en la irregularidad de las tábulas presentes en la zona axial del nuevo taxón. Por otro lado, los cálices de Parastriatopora son poco profundos y aunque, en algunas especies, están ornamentados por arrugas septales similares a las apreciadas en Argentinella, éstas no muestran alineamientos de gránulos y sí depresiones puntiformes, más o menos marcadas, en la franja de contacto entre la pared y el fondo calicinal, las cuales no han sido observadas en el género argentino.

El material tipo de Crenulipora Le Maître ha sido recientemente revisado por Fernández-Martínez et al. (1999b). Este género norteafricano comparte con Argentinella nov. gen. la naturaleza microlamelar de la pared, el fuerte desarrollo del aparato septal constituido por múltiples espinas y la irregularidad de las tábulas. No obstante, la pared de Crenulipora no muestra un engrosamiento periférico marcado y su lámina media es sinuosa mientras que en Argentinella se muestra 

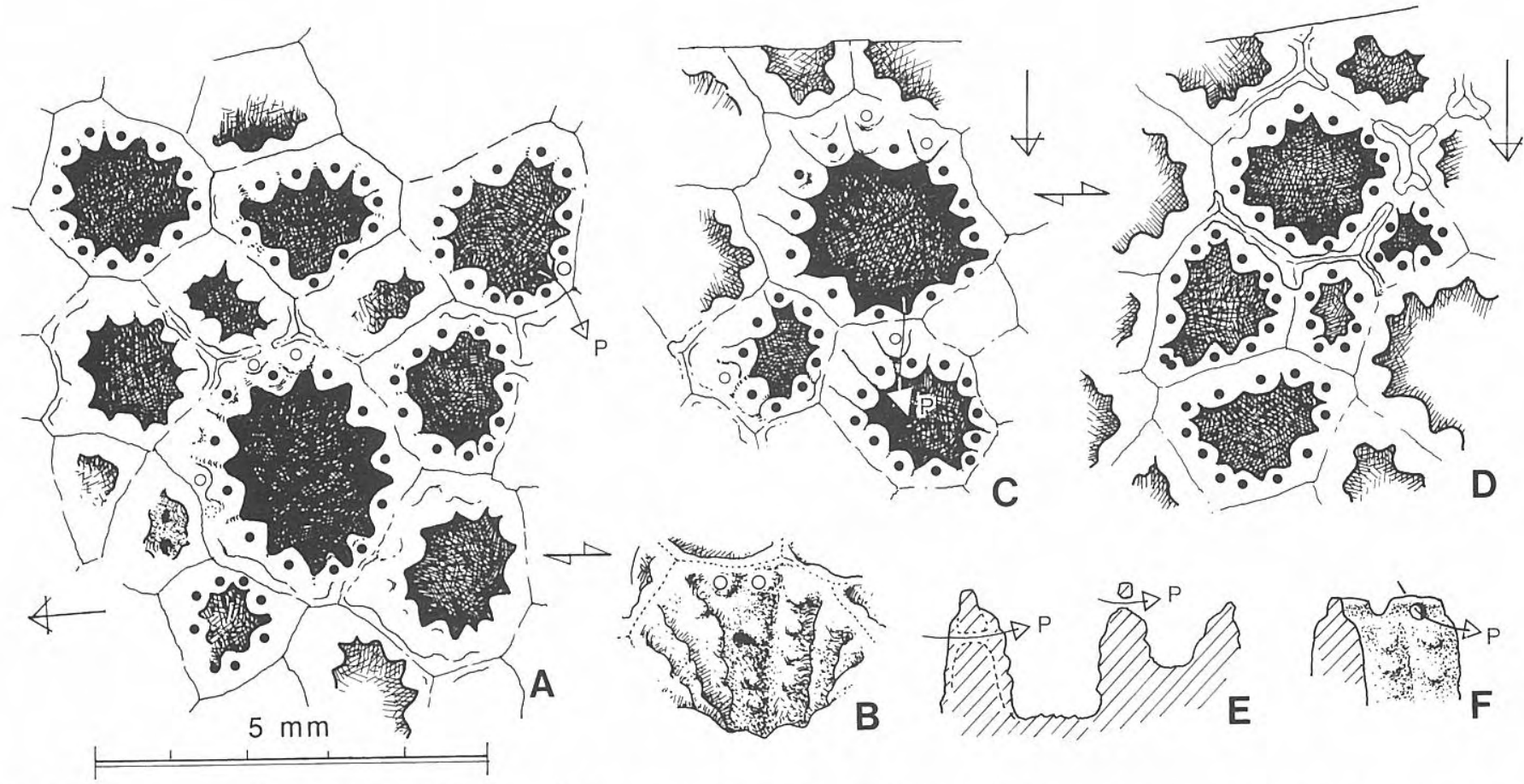

Figura 1.Argentinella argentina (Thomas, 1905), morfología de los cálices. Dibujos semi-esquemáticos que muestran las arrugas septales (indicadas individualmente con un circulo negro) y su ramificación distal (círculos vacíos), así como el emplazamiento de algunos poros P1 o P2 indiferenciados (P); la flecha asociada a cada dibujo indica el eje y la dirección de crecimiento de la rama. Los cálices donde se indica el número de arrugas septales se leen siempre de izquierda a derecha y de arriba a abajo de la figura. A. Ejemplar IMGPGö 495-100, lectotipo. Puede observarse el siguiente número de arrugas septales: 12; 10 o más de 10; 12 (una de ellas es una arruga doble en las proximidades de un poro); 11; 12 (entre las cuales hay una arruga doble neta en la parte superior y otra dudosa a la izquierda); $7 \mathrm{o}$ más de 7. B. Ejemplar IMGPGö 495-100, lectotipo. Vista oblicua del cáliz central de la figura A, mostrando el desdoblamiento distal de la fila de gránulos/espinas que ornamentan una arruga. Notar igualmente la presencia de un poro P2 sobre esta misma arruga. C. Ejemplar IMGPGö 495-101, paralectotipo. Número de arrugas septales 12 (una doble); 9 o 10?; 12 (una de ellas modificada y situada sobre un poro P2). D. Ejemplar IMGPGö 491-101, paralectotipo. Número de arrugas septales: 12; 6 o más de $6 ; 12$ ?; 7; 12. Observar la presencia de una cresta media bastante bien desarrollada en este sector de la rama. E. Esquema de una sección axial de los cálices, mostrando la presencia de un poro entre las arrugas septales y en la base de la cresta media. F. Esquema de la cresta media con una depresión generada por un poro incompleto y otro poro completo (P).

Morphology of the calices. Schematic sketches showing septal ridges (individually represented by a black circle) and their distal bifurcation (represented by white circles), as well as the positioning of some indifferentiated pores (PI or $P 2$, represented in the diagram by $P$ ). The arrow which appears in each diagram indicates the axis and the direction of growth of the branch. Where the number of septal ridges of each calice is indicated, they should be read from left to right, and from the top to the bottom of the figure. A. Sample IMGPGö 495-100, lectotype. The following number of septal ridges can be observed: 12; 10 or more than 10; 12 (one of these is a double ridge near a pore); 11; 12 (amongst which there is a clear double ridge at the top and another, less clear, on the left); 7 or more than 7 . B. Sample IMGPGö 495-100, lectotype. Oblique view of the central calice in the figure A, showing the distal division of the row of granulae/spines on each ridge. Note also the presence of a P2 pore on this same ridge. C. Sample IMGPGö 495-101, paralectotype. Number of septal ridges: 12 (one double); 9 or 10?; 12 (one modified and positioned over a P2 pore). D. Sample IMGPGö 491-101, paralectotype. Number of septal ridges: 12; 6 or more than 6; 12?; 7; 12. Note the presence of a fairly well developed central crest in this section of the branch.E. Axial section diagram of the calices, showing the presence of a pore in the septal ridges and again at the base of the central crest. $F$. Diagram of the central crest with a depression generated by an incomplete pore and another, complete, one $(P)$.

rectilínea; además, sus cálices carecen de arrugas septales y las espinas calicinales, muy abundantes, se reparten de forma irregular y no en alineamientos, como ocurre en el taxón argentino. Otra posible diferencia de interés es la presencia, en Crenulipora, de una cresta cortante y crenulada que limita los cálices, cuya morfología es diferente a la de la cresta observada de forma local en Argentinella.

Striatopora Hall es un género tradicionalmente usado como comodín y que demanda revisión. Para su comparación con Argentinella nos basaremos en la descripción y figuración de su especie tipo, S. flexuosa 

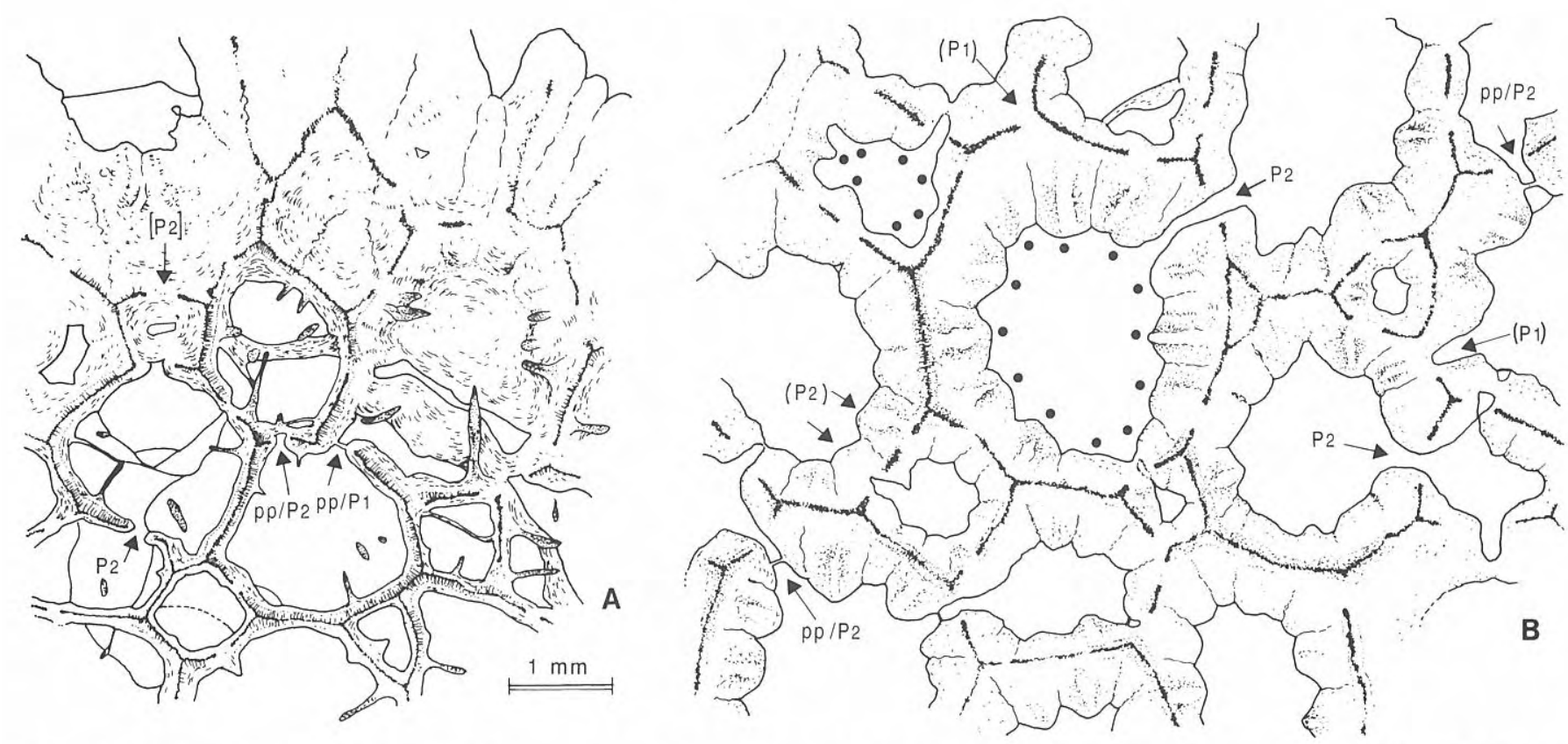

Figura 2.Argentinella argentina (Thomas, 1905), tipos y reparto de los poros en la pared. Ejemplar IMGPGö 491-98, paralectotipo dibujado a partir de las láminas originales de Thomas. A (sección figurada por Thomas, 1905 en la parte superior de la página 268), sección transversal, límite zona axial-zona periférica; en ella se han representado también los elementos microestructurales (fibroides en contacto con la lámina media, esclerénquima microlamelarlamelar y espinas). B (sección figurada por Thomas, 1905 en la parte media de la página 268), sección tangencial. El fuerte espesor de esta lámina delgada impide realizar observaciones microestructurales, sin embargo las arrugas septales se identifican claramente $(7,12)$ y se observa la silueta de una espina en el plano medio de cada arruga. P1 = poro angular; P2 = poro mural; pp = diafragma; siglas entre paréntesis = la sección pasa por encima o por debajo del poro; siglas entre corchetes = obturación secundaria del poro por esclerénquima mural.

Type and distribution of wall pores. Sample IMGPG̈̈ 491-98. Paralectotype diagram based on the original plates made by Thomas. A (section figured by Thomas, 1905 at the top of $p$. 268), cross section, axial-peripheral zone border; microstructural elements ("fibroids" touching the central plate, microlamellar-lamellar sclerenchyme, and spines). B (section figured by Thomas, 1905 in the middle of p. 268), tangential section. The pronounced thickness of this thin section prevents microstructural examination, however the septal ridges can be clearly identified (7, 12) and the shadow of a spine in the middle of each ridge is observed. $P 1=$ angular pore; $P 2=$ wall pore; pp $=$ pore plate; symbols in brackets () mean that the section is taken from above or below the pore; symbols in brackets [] signify secondary filling of the pore by wall sclerenchyme.

Hall, realizadas por Oliver (1966). Striatopora comparte con el taxón sudamericano varios rasgos: la microestructura microlamelar; la morfología externa cilíndrica y el engrosamiento periférico de la pared. A pesar de estas similitudes, ambos géneros se diferencian claramente por la presencia, en Striatopora, de cálices fundamentalmente asimétricos separados por una cresta media con forma de escudo, dos tipos de coralitas distintas, las tábulas regulares y el escaso desarrollo del aparato septal. Cabe añadir que, en Striatopora, también han sido descritas 12 crestas pero parece tratarse, básicamente, de estructuras internas; además, no hay datos precisos sobre la naturaleza de sus poros, por lo que no puede realizarse una comparación sobre estos dos últimos elementos.

Otro género próximo al nuevo taxón es Kolymopora Preobrazhensky cuyos cálices tienen 12 espinas septales muy bien marcadas pero que desarrolla tábulas simples y completas y donde no ha sido citada la presencia de poros angulares. La microestructura de este género ha sido descrita como trabecular, aunque el material estudiado por los autores de este trabajo y procedente del Ordovícico de China muestra una típica microestructura microlamelar.

Por tanto, y a pesar de la existencia de taxones con microestructura y morfología, (tanto a nivel de elementos externos como internos) similares, el material procedente del Lochkoviense del Cerro del Fuerte y descrito por Thomas en 1905 no puede incluirse adecuadamente en ninguno de ellos. Por este motivo, se crea con dicho material el nuevo género Argentinella que, por el momento, acoge exclusivamente a la especie Argentinella argentina (Thomas, 1905).

\section{Especies incluidas}

Solamente la especie tipo.

\section{Argentinella argentina (Thomas, 1905)} Figs. 1-8

Localidad típica: Cerro del Fuerte, Precordillera Argentina. 


\begin{tabular}{||c|c|c|c|c|c|c||}
\hline \hline $\mathrm{N}^{0}$ lados & 9 & 8 & 7 & 6 & 5 & 4 \\
\hline Rango (N) & $2,6-3,5(6)$ & $2,5-3,1(6)$ & $2-3,5(12)$ & $1,8-3,3(17)$ & $1,3-2,5(16)$ & $0,7-1,6(9)$ \\
\hline Media arit. & 3,3 & 2,7 & 2,6 & 2,4 & 1,8 & 1,2 \\
\hline \hline
\end{tabular}

Tabla 1. Medidas (en mm) del diámetro de los cálices en el lectotipo, expresadas en función del número de lados. En cada columna (de 4 a 9 lados), se indica el rango, número de medidas realizadas (N) y la media aritmética de las mismas. Diameter measurement (in $\mathrm{mm}$ ) of the lectotype calices, expressed in terms of side number. For each column (from 4 to 9 sides), range, number of measurements $(N)$ and the arithmetical median of these, is given.

Estrato típico: Lochkoviense. Posiblemente niveles superiores de la Fm. Los Espejos.

\section{Material}

Material tipo: Lectotipo designado en este trabajo, IMGPGö 495-100 (ejemplar figurado por Thomas, 1905 en Lám. XII, fig. 20a; del cual se han realizado tres láminas delgadas, dos acetatos y cuatro láminas ultrafinas del tipo LFP: tres antiguas realizadas por J. Lafuste y nominadas FA1-3 y una reciente signada B 47044). Paralectotipos, IMGPGö 495-98 (únicamente constituido por tres láminas delgadas antiguas, figuradas por Thomas, 1905, en la página 268); IMGPGö 495-99 (ejemplar figurado por Thomas (op. cit.), en Lám. XII, fig. 20 y 20b; del cual se han realizado dos láminas delgadas) y IMGPGö 495-101 del cual se han realizado dos acetatos. Es posible que las tres láminas antiguas que portan la indicación 495-98 procedan del ejemplar 495-101 pero ha sido imposible comprobarlo con precisión.

Otro material (topotipos): 15 ramas, de las cuales se han realizado 9 láminas delgadas, conteniendo diversas secciones transversales y longitudinales así como 7 láminas del tipo LFP y 7 acetatos correspondientes a éstas. Este material se encuentra signado como IMGPö 495-102 a IMGPö 495-116.

Además, han sido realizadas dos series de moldes y acetatos. Una de ellas se encuentra depositada en la colección de la Universidad de Bretaña Occidental con las siglas LPB 12.488 y la otra en la colección de la Universidad de Oviedo bajo la signatura DPO 15.445.

\section{Diagnosis}

Ramas de Argentinella de tamaño entre 0,7 y $2 \mathrm{~cm}$, constituidas por cálices poligonales, los adultos con diámetros entre 1,8 y 3,5 mm. Internamente, zona axial y periféricas mal delimitadas. En la zona axial, coralitas mayores con tamaños entre 1,5 y 2,5 mm; pared de espesor en torno a $0,12-0,35 \mathrm{~mm}$; poros con diámetros de 0,15 $\mathrm{mm}$; tábulas irregulares a verticales, con distancias muy variables. En la zona periférica, coralitas de tamaños entre 2 y $3,5 \mathrm{~mm}$; pared de grosor entre 0,35 y $1 \mathrm{~mm}$; poros frecuentes, de diámetros entre 0,15 y $0,2 \mathrm{~mm}$; tábulas regulares, situadas a distancias entre 0,4 y $0,75 \mathrm{~mm}$.

\section{Descripción}

Morfología externa

El material corresponde a fragmentos de ramas, en su mayoría irregulares por engrosamientos globosos, excrecencias o desarrollo ocasionalmente curvado. El ejemplar de mayor tamaño (lectotipo) mide $4,5 \mathrm{~cm}$ de longitud; la anchura de las ramas oscila entre 0,7 y $2 \mathrm{~cm}$ y una misma rama puede tener contorno cilíndrico u oval en diferentes puntos. En cinco ejemplares, incluido el tipo, se aprecian indicios de bifurcación dicotómica, en un ángulo variable que puede ser incluso perpendicular a la dirección de la rama (ejemplar IMGPGö 495-105). El ejemplar IMGPGÖ 495-99 muestra una zona engrosada con desarrollo incipiente de cuatro ramas.

En los ejemplares IMGPGö 495-99, IMGPGö 495-102 y IMGPGö 495-103 se observa la presencia muy local de bandas constituidas por tejido esquelético liso o con estrías epitecales, que cierran las aperturas de los cálices. El mismo aspecto se observa también en la pared de una excrecencia lateral que aparece en el ejemplar tipo, aunque, en este último caso, es difícil reconocer si se trata o no de parte de la colonia.

Figura 3.Argentinella argentina (Thomas, 1905), estructura del esqueleto. Dibujos a cámara clara de láminas delgadas de espesor petrográfico salvo mención en contra. A-C, ejemplar IMGPGö 495-100, lectotipo. A, sección transversal distal en la base de la ramificación de la rama, acetato realizado sobre lámina delgada gruesa; en la zona periférica (flechas) observar las espinas dispuestas en una fila única en el plano medio de las arrugas septales. B, sección transversal proximal. C, sección longitudinal axial a subaxial. D. Ejemplar IMGPGö 495-101, paralectotipo. Sección transversal, dibujo realizado a partir de un acetato (que no permite una buena observación de la lámina media). E, F. Ejemplar IMGPGö 495-107. Sección transversal y longitudinal. Observar una zona de necrosis y recrecimiento posterior (flecha).

Skeletal structure. Clear chamber diagram of thin sections with petrographic thickness, except when specified to the contrary. A-C, sample IMGPGö 495-100, lectotype. A, distal cross section at the base of the branch bifurcation, peel made of a thick thin section; note in the peripheral zone the presence of a single row of spines positioned in the middle of the septal ridges. B, proximal cross section. C, axial to subaxial longitudinal section. D. Sample IMGPGö 495-101, paralectotype. Cross section, drawing based on a peel (which prevents a detailed examination of the section). E, F. Sample IMGPGö 495-107. Cross and longitudinal section. Note an area of necrosis and subsequent regrowth (arrow). 


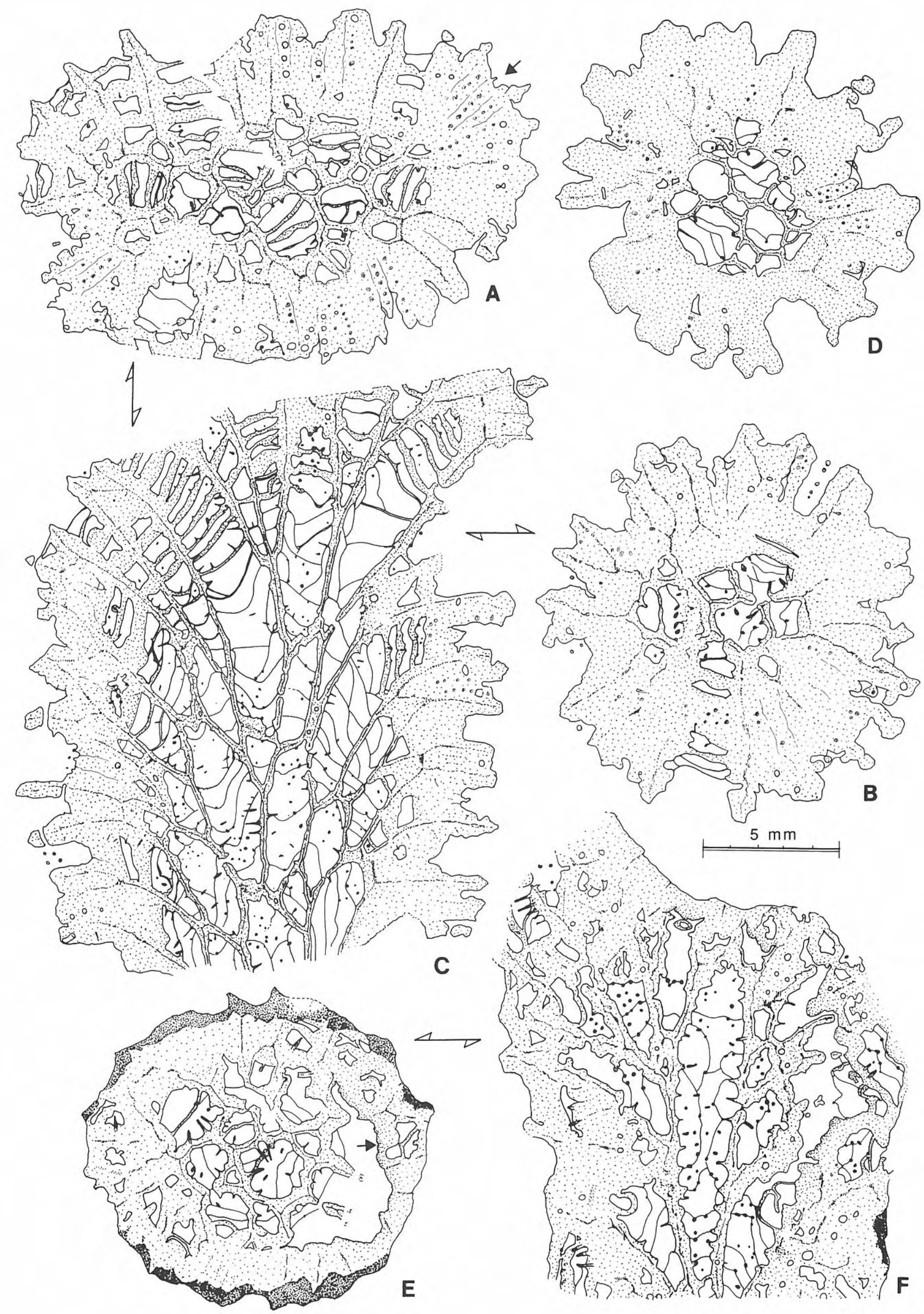



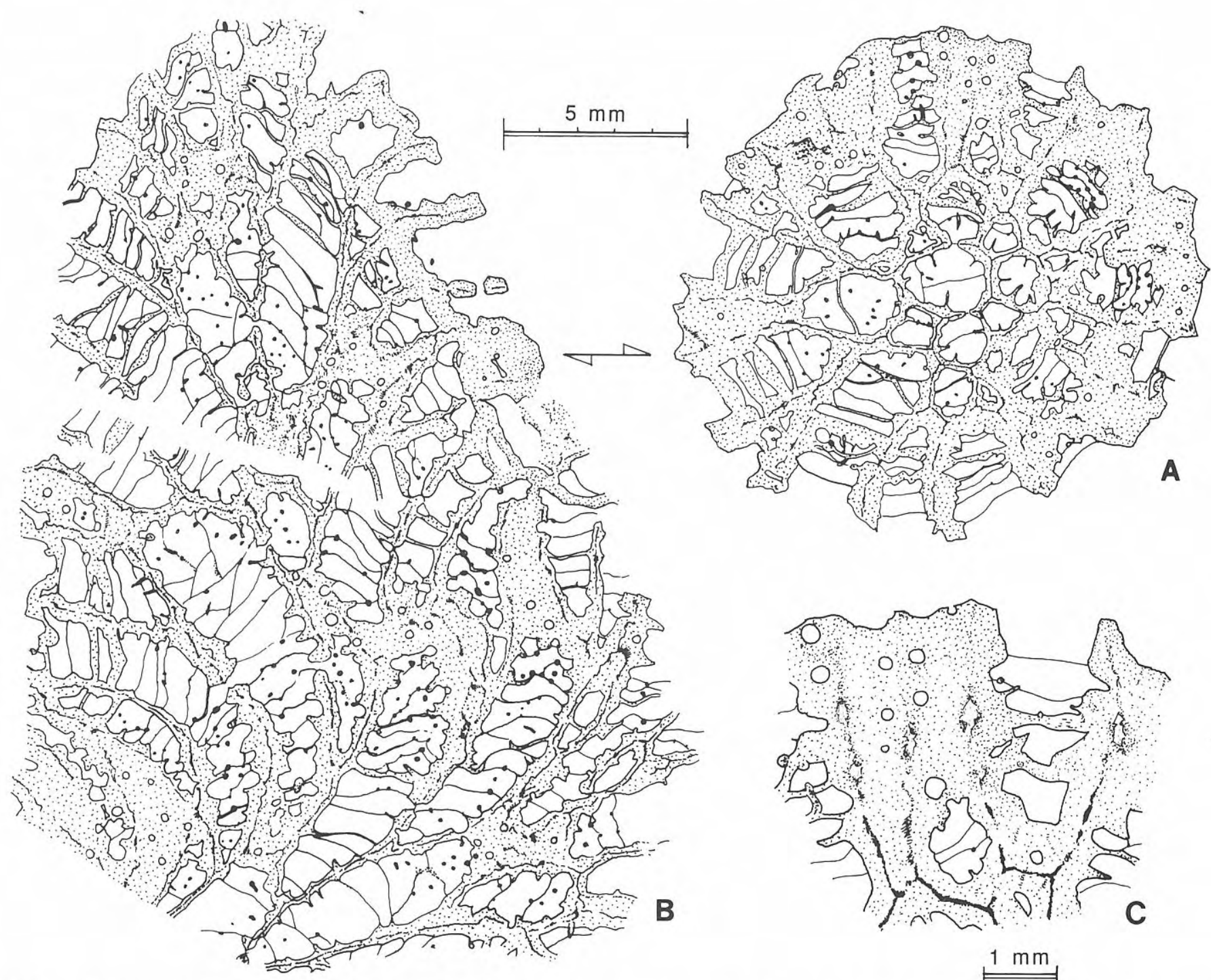

Figura 4.Argentinella argentina (Thomas, 1905), estructura del esqueleto y aspecto microestructural parcial, ejemplar IMGPGö 495-102. A, sección transversal. B, sección longitudinal. C, detalle de A mostrando, en la zona periférica, el reemplazamiento de la lámina media por elementos claros de diseño más o menos losángico.

Skeletal structure and partial microstructural feature, sample IMGPGö 495-102. A, cross section. B, longitudinal section. $C$, detail of $A$, showing, in the peripheral zone, the replacement of the median suture by clearly outlined, more or less losange-shaped elements.

\begin{tabular}{||l|c|c|c|c||}
\hline \hline EJEMPLAR & Dc (8-9 lados) & Dc (5-7 lados) & Dc (3-4 lados) & Dc (zp) \\
\hline Lectotipo & $2,2-2,4(3)$ & $0,9-1,85(10)$ & $0,65-1,2(7)$ & $2,8-1,9(7)$ \\
\hline Para-y Topotipos & $1,5-2,5(8)$ & $0,8-2,25(18)$ & $0,65-1,35(8)$ & $2,1-3,4(8)$ \\
\hline
\end{tabular}

Tabla 2. Medidas (en mm) del diámetro de las coralitas (Dc) en el lectotipo y en los demás ejemplares (paralectotipos y topotipos) estudiados. Las primeras casillas expresan estas medidas en función del número de lados y contabilizada de lámina media a lámina media; la última casilla contiene los diámetros interiores de las coralitas en la zona de desembocadura. En cada columna se indica el rango y número de datos obtenidos. Dc = diámetro de las coralitas; zp = zona periférica.

Diameter measurements ( $D c$ ) (from median suture to mediam suture, in $\mathrm{mm}$ ) of the corallites in all of the samples studied (lectotype, paralectotypes and topotypes). The first columns give these data in terms of side number; the last column contains corallite diameter for the opening zone. In each column the range and number of data obtained is indicated. $D c=$ corallite diameter; $z p=$ peripheral $z$ one. 


\begin{tabular}{|l|c|c|c|c|c|c||}
\hline \hline EJEMPLAR & Gpa (za) & Gpa (zp) & Dpo (za) & Dpo (zp) & Dst tab (za) & Dst tab (zp) \\
\hline Lectotipo & $\mathbf{0 , 1 2 - 0 , 1 8}$ & $\mathbf{0 , 4 0 - 1}$ & $\mathbf{0 , 1 5}$ & $\mathbf{0 , 1 5 - 0 , 2}$ & $\mathbf{0 , 5 - 1 , 6}$ & $\mathbf{0 , 6 - 0 , 7}$ \\
\hline Para- y Topotipos & $\mathbf{0 , 1 2 - 0 , 3 5}$ & $\mathbf{0 , 3 5 - 1}$ & $\mathbf{0 , 1 5 - 0 , 1 7}$ & $\mathbf{0 , 1 5 - 0 , 2}$ & - & $\mathbf{0 , 4 - 0 , 7 5}$ \\
\hline
\end{tabular}

Tabla 3. Datos cuantitativos (expresados en $\mathrm{mm}$ ) de pared, poros y tábulas para el lectotipo y resto de ejemplares (paralectotipos y topotipos) estudiados. Gpa = espesor de la pared; Dpo = diámetro de los poros; Dst tab = distancia entre tábulas; $\mathrm{za}=$ zona axial; $\mathrm{zp}=$ zona periférica.

Quantative data (in mm) for wall thickness, pores and tabulae in all of the samples studied (lectotype, paralectotypes and topotypes). Gpa = wall thickness; Dpo = pore diameter; Dst Tab = distance between tabulae; za = axial zone; $z p=$ peripheral zone.

Las ramas están constituidas por cálices poligonales (Fig. 1), desiguales en forma y tamaño, con frecuencia ligeramente estirados en dirección transversal, muy profundos y con desembocadura perpendicular a la superficie de la colonia. Están delimitados por un borde saliente y crenulado, que con frecuencia se ensancha hasta formar una estrecha plataforma constituida internamente por 10-12 arrugas septales (Fig. 1A, C, D). Estas arrugas se encuentran ornamentadas por alineamientos, regularmente espaciados, de espinas groseras, muchas veces con aspecto de gránulos y que frecuentemente destacan en el borde interno de la pared. En algunos cálices, la fila de espinas o gránulos que define distalmente cada arruga se desdobla en dos alineamientos, generándose así la impresión de que existen 13 crestas en dicho cáliz (Fig. 1A-C).

Los cálices más jóvenes (con 4-5 lados) son menos profundos y más o menos cónicos, es decir, carentes en algunos casos de un fondo calicinal bien desarrollado, pero la ornamentación basada en alineamientos de espinas está ya presente en ellos.

Entre las arrugas septales y sobre ellas se aprecia el desarrollo local de poros de contorno circular y en número y disposición variable. Así, se han observado tanto poros de tipo P1 (situados en los ángulos) como P2 (desarrollados en la pared) (Fig. 2) aunque, en láminas delgadas, y si no se observa claramente la traza de la lámina media, resulta difícil reconocer el tipo exacto de poro (Fig. 1B, E, F).

En algunos ejemplares (IMGPGÖ 495-100 y 495-101), la zona de la lámina media que separa dos cálices está ocupada por una pequeña cresta, muy estrecha y también horadada por poros (Fig. 1E-F). Es posible que esta cresta media se encontrara, originariamente, más desarrollada y que no sea frecuente debido a su fragilidad ante la erosión.

Los fondos de los cálices, con frecuencia muy profundos, están ocupados por tábulas planas de superficie ligeramente granulosa.

Los cálices descritos, profundos y con un fuerte desarrollo del aparato septal, se aprecian especialmente bien en el ejemplar seleccionado como lectotipo, así como en ramas bien conservadas y en las zonas menos deterioradas de varias muestras. Otros ejemplares se encuentran alterados, erosionados o parcialmente embebidos en matriz, por lo que no puede observarse ni la profundidad ni la ornamentación de los cálices, aunque localmente aparecen indicios de ambos rasgos.

Tan sólo de forma puntual, en ramas de crecimiento incipiente, se aprecia la presencia de cálices oblicuos, con desarrollo de plataformas superiores que pueden aparecer punteadas de espinas y poros.

El tamaño de los cálices en el ejemplar tipo puede consultarse en la Tabla 1. En el resto de los ejemplares estudiados, los cálices muestran tamaños similares a los aquí anotados.

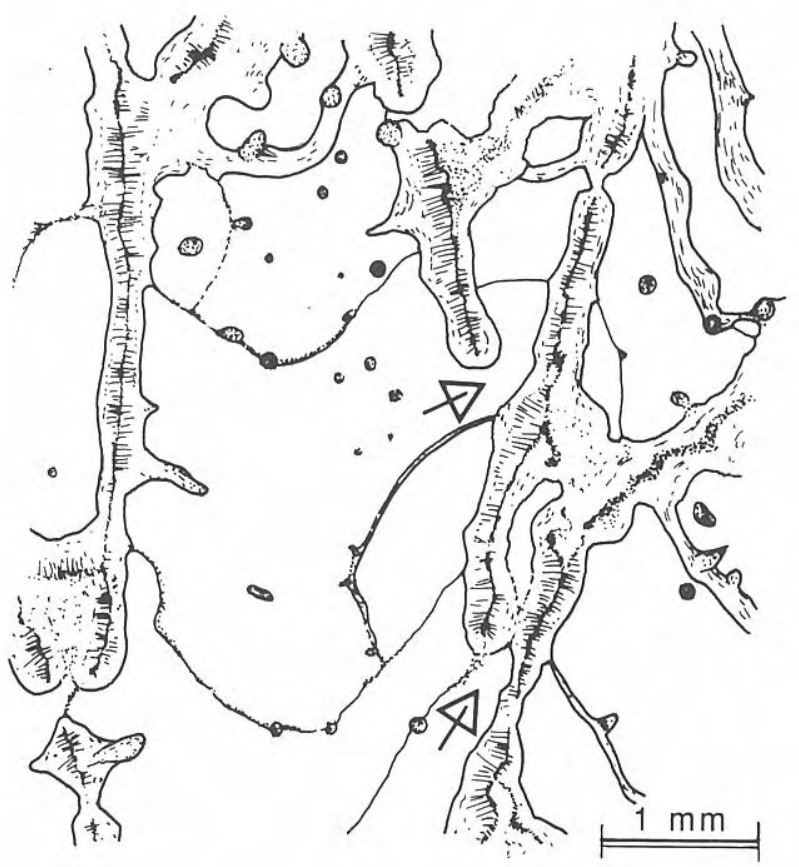

Figura 5.Argentinella argentina (Thomas, 1905), gemación. Ejemplar IMGPGö 495-98, paralectotipo, detalle de la lámina figurada por Thomas, 1905, sección longitudinal de la página 268. Figuras de gemación lateral en la parte dorsal de la coralita madre. Las flechas indican el emplazamiento del poro basal (que en la parte inferior no aparece bien definido por estar seccionado lateralmente al mismo).

Budding. Sample IMGPGö 495-98, paralectotype, detail of the plate figured by Thomas, 1905, longitudinal section from p. 268. Figures showing two lateral buddings along the back of the original corallite. Arrows indicate the position of the basal pore (which is not well defined in the lower part because of lateral sectioning of itself). 

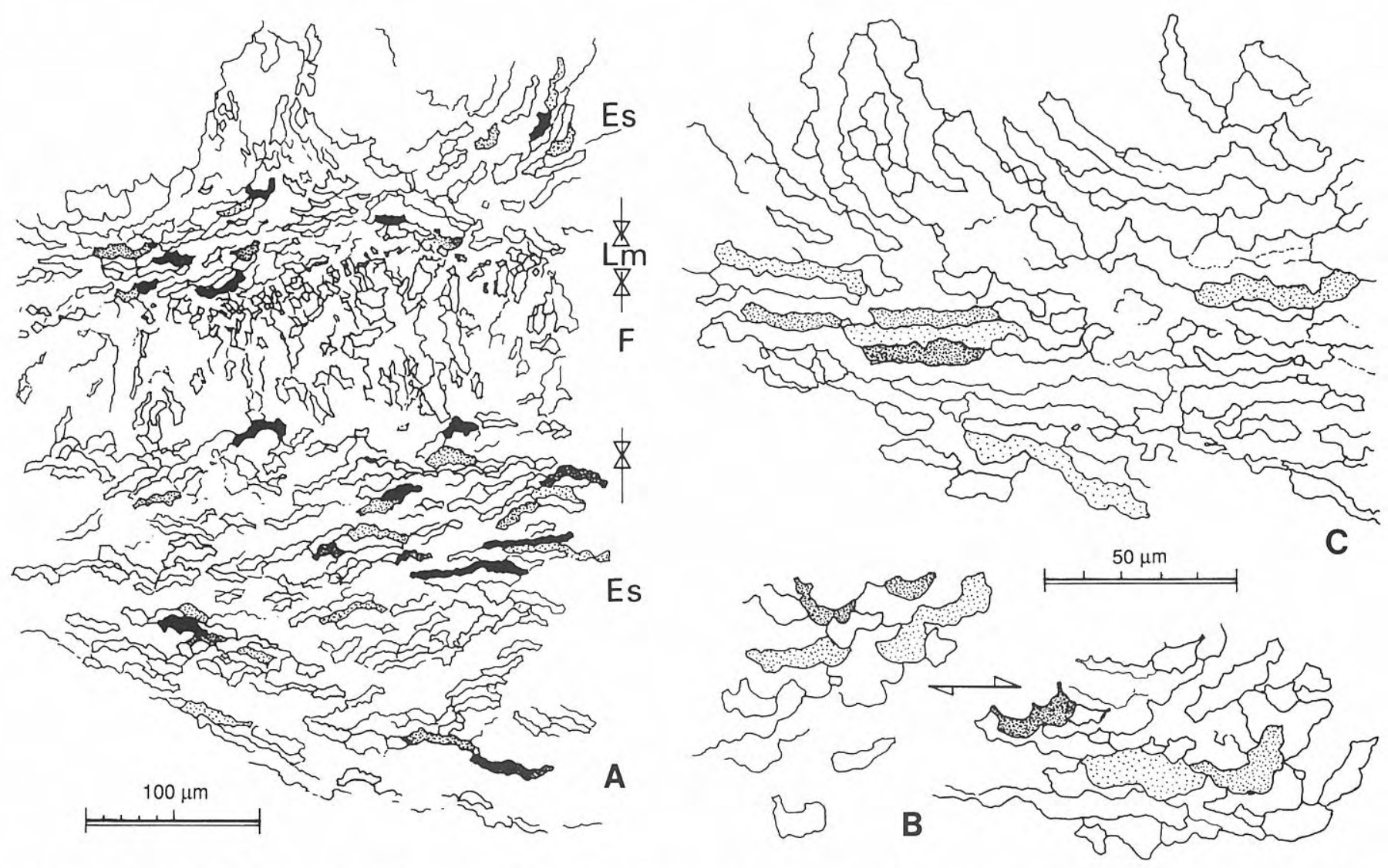

Figura 6. Argentinella argentina (Thomas, 1905), microestructura de la pared. A, ejemplar IMGPGö 495-107, lámina LFP B 44168, sección transversal, zona axial. Lm = lámina media granular; F = fibroides, en este caso sólo desarrollados en uno de los lados de la lámina media; Es = esclerénquima de la pared constituido por microlamelas y lamelas. B, ejemplar IMGPGö 495-108, lámina LFP B 44166, sección longitudinal, límite zona axial-zona periférica. Microlamelas. C, ejemplar IMGPGö 495-110, lámina LFP B 44170, sección tangencial, límite zona periférica. Lamelas.

Wall microstructure. A, sample IMGPGö 495-107, ultra-thin section LFP B 44168, cross section, axial zone. Lm= granular median suture; $F=$ fibroids, in this case only developed on one side of the median suture; Es = wall sclerenchyme consisting of microlamellae and lamellae. B, sample IMGPG̈̈ 495-108, ultra-thin section LFP B 44166, longitudinal section, peripheral axial zone border. Microlamellae. C, sample IMGPG̈̈ 495-110, ultra-thin section LFP B 44170, tangential section, peripheral zone border. Lamellae.

\section{Morfología interna}

Observaciones a la descripción morfológica interna: Es importante señalar que, a pesar de que el número de ejemplares estudiados es moderadamente alto (12 ramas en el caso de la morfología interna), no ha podido realizarse, por varias causas que a continuación se exponen, ningún estudio cuantitativo con base estadística válida sobre los rasgos morfológicos internos.

En el caso de los diámetros de las coralitas, esto es debido a que las secciones transversales de las ramas contienen muy pocas (en algunos casos, ninguna) coralitas seccionadas de forma no tangencial, por lo que las dimensiones medidas son aleatorias; además, el número de coralitas por sección no es alto.

La distancia entre tábulas es un rasgo altamente variable y su determinación está dificultada por la irregularidad de su disposición; por otro lado, en las zonas de transición y periférica, las tábulas pueden aparecer recubiertas por una capa más o menos espesa de esclerénquima cuyo desarrollo no parece atender a ningún patrón fijo. En todos los casos, la distancia entre tábulas se ha medido de base de una tábula a base de la siguiente y en las zonas medias de las coralitas.

En este marco, tan solo han podido cuantificarse con cierta precisión el diámetro de los poros y algunos rasgos de las espinas (por otro lado, también muy variables), pero ninguno de estos dos caracteres es definitivo en las diagnosis a nivel específico de los corales tabulados.

Ante esta problemática, se ha optado por realizar el mayor número de medidas posibles en diferentes situaciones y por reflejar estos datos en la descripción morfológica y en las Tablas 2 y 3 .

\section{Coralitas}

En sección transversal, las ramas aparecen formadas por una zona axial rodeada de una corona periférica (Fig. 3B, D, E; Fig. 4A). La zona limítrofe está ocupada por secciones tangenciales o longitudinales de coralitas y puede llegar a ser muy amplia, por lo que no suele existir una delimitación neta 

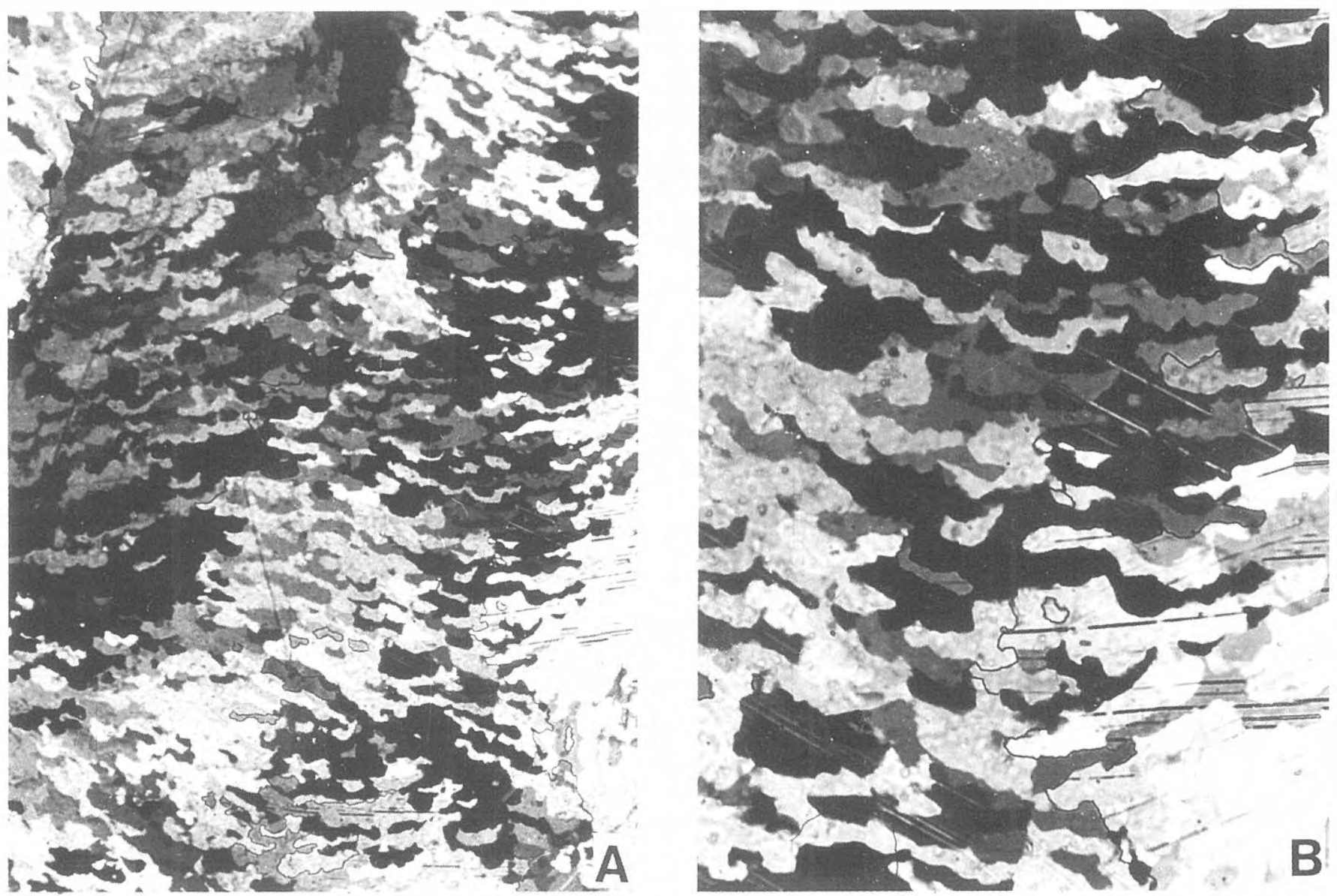

Figura 7.Argentinella argentina (Thomas, 1905), microestructura de la pared. Ejemplar IMGPGö 495-100, lectotipo, lámina LFP B 47044, sección transversal, zona periférica, microlamelas. Crecimiento del esqueleto hacia arriba. A, obsérvese el ligero levantamiento hacia atrás de las microlamelas en el contacto de una espina (sombra oscura en la parte superior de la foto) situada en el plano medio de una arruga septal. x250. B, morfología de las microlamelas, sector medio derecho de A, x625.

Wall microstructure. Sample IMGPGö 495-100, lectotype, ultra-thin section LFP B 47044, cross section, peripheral zone, microlamellae. Upward growth of the skeleton. A, note the slight backward rising of the microlamellae in contact with a spine (dark shadow at the top of the photograph) which is situated in the middle of a septal ridge. $x 250 . \mathrm{B}$, microlamellae morphology, from the middle right section of $A, x 625$.

entre región central y corona periférica. En la zona axial, el número de secciones transversales de coralitas es muy escaso (frecuentemente sólo 1 o 2) y aparecen rodeadas por las secciones tangenciales de las coralitas que se encuentran en fase de curvatura. En conjunto y aunque es muy variable, suele haber 1 o 2 coralitas gerónticas ( 8 lados o más), junto a 2-4 adultas (5 a 7 lados) y alguna juvenil (3-4 lados).

Las medidas del diámetro, realizadas de lámina media a lámina media en las secciones transversales y en algunas tangenciales (en este caso en la dirección perpendicular al crecimiento de la coralita) se indican en la Tabla 2, donde también aparecen algunas medidas realizadas en la zona periférica, es decir, en los cálices. El aumento del tamaño de las coralitas hacia la zona periférica es muy neto y tiene lugar ya desde la zona axial, continuándose de forma progresiva hasta la perpendicularización de las mismas y, en algunas ocasiones, algo más allá.

La generación de nuevas coralitas se produce por brote lateral, que acontece en la región dorsal de la coralita madre y está asociada a un poro basal (Fig. 5).
Poros

Los poros pueden ser de tipo P1 (situados en los ángulos) o de tipo P2 (situados sobre la pared, generalmente en la parte media de la misma) (Fig. 2). Algunos de ellos se encuentran cerrados por diafragmas. En varias secciones se observa su contorno redondeado y su alineamiento en una fila (Fig. $3 \mathrm{C}$ ), con una separación entre ellos que oscila entre 0,15 y $0,20 \mathrm{~mm}$. Su diámetro en la zona axial se mantiene bastante constante en torno a $0,15 \mathrm{~mm}$, mientras que en periferia se han medido diámetros próximos a los 0,17-0,20 mm. El número de poros que aparece en las secciones de los diferentes ejemplares es muy variable pero, en general, se trata de un elemento muy usual, especialmente en las regiones calicinales de las coralitas.

\section{Aparato septal}

El aparato septal está constituido por espinas muy abundantes y repartidas por toda la colonia, que se observan bien como elementos espinosos desarrollados en la pared, bien en forma de múltiples secciones circulares flotando en 
los lúmenes de las coralitas (ver, entre otras, Figs. 2, 3 y 5). En general, se trata de espinas rectas y largas (longitudes medidas entre 0,35 y $0,55 \mathrm{~mm}$ aunque se ha observado el caso de una espina que tiene un recorrido de $1 \mathrm{~mm}$ ), de sección usualmente circular (diámetros entre 0,15 y 0,20 $\mathrm{mm}$ ), que se disponen en 1 o 2 filas sobre la pared, manteniendo distancias, en ocasiones muy cortas, de 0,1 a $0,2 \mathrm{~mm}$.

En los ejemplares con mayor número de espinas, éstas se sitúan localmente al mismo nivel y pueden existir hasta 3 filas por cara. También en estas ramas con aparato septal muy desarrollado son frecuentes las espinas dirigidas hacia arriba, a la manera de las que caracterizan el género Astrocerium.

Como se indicó en el apartado de Morfología externa, la observación de los cálices permite apreciar estas espinas en las coralitas adultas como elementos muy engrosados y dispuestas en alineamientos precisos que recorren el plano medio de las arrugas septales (Fig. 3A).

\section{Tábulas}

En la zona axial, las tábulas son delgadas y oscuras, similares en estructura a la lámina media. En cambio, hacia la periferia, muchas de estas tábulas aparecen recubiertas de esclerénquima microlamelar desarrollado en continuidad con el de la pared. Este engrosamiento suele ser coincidente con el inicio de la curvatura de las coralitas, pero no siempre ocurre así ni tampoco acontece de una forma continua y progresiva, ya que muchas tábulas periféricas están desprovistas del mismo o lo tienen menos desarrollado que otras tábulas anteriores (Fig. 3C, F).

La morfología de las tábulas es también muy variable aunque, en general, las de la zona axial son más irregulares que las presentes en regiones periféricas. Así, son frecuentes las tábulas axiales incompletas (Fig. 3C), colgadas o de desarrollo no horizontal. Varias ramas contienen en su región central tábulas muy inclinadas e, incluso, totalmente verticales (Fig. 3F), en ocasiones asociadas a otras de desarrollo similar generando así un aspecto claramente vesiculoso. En otros casos, esta irregularidad se limita a recorridos no horizontales, con fuertes depresiones, generalmente en la zona central de la tábula.

En las regiones de inicio de curvatura de las coralitas, las tábulas tienen recorridos más rectos, pueden ser horizontales o ligeramente inclinadas y en ellas se han observado varios casos aislados de tábulas incompletas apoyadas en otras (Fig. 3C, especialmente sector derecho de la rama). En la zona periférica, el desarrollo de estos elementos es más regular, aunque tanto las zonas basales como cuspidales de muchas tábulas engrosadas muestran pequeñas irregularidades locales.

La distancia entre tábulas consecutivas es también muy variable. En la zona axial, y debido a la irregularidad antes mencionada, resulta difícil realizar medidas aunque puede señalarse que las distancias apreciadas varían entre 0,5 y 1,6 $\mathrm{mm}$. En la zona periférica aparecen bastante más juntas habiéndose medido distancias entre 0,4 y $0,75 \mathrm{~mm}$.

Por regla general, tábulas y espinas no se encuentran relacionadas pero la abundancia de ambos elementos genera intersecciones entre ellos y, así, es fácil observar tábulas apoyadas, recortadas o deformadas por espinas (Fig. 3C, F y Fig. 5). Más frecuente, sobre todo en los ejemplares con mayor desarrollo del aparato septal, es la presencia de espinas tabulares que aparecen en número variable sobre determinadas tábulas (Fig. 3C). En general se trata de pequeñas espinas dirigidas hacia arriba, bien rectas o

Figura 8. Argentinella argentina (Thomas, 1905), Cerro del Fuerte, Lochkoviense. Lectotipo y topotipos. a, c, f. Ejemplar IMGPGö 495-100 figurado por Thomas (1905, Lám. XII, fig. 20a). Lectotipo. a. Vista general de la rama antes de ser seccionada para la realización de láminas delgadas, mostrando una dicotomía distal (triangulos), x2. c. Morfología de los cálices, x5. f. Sección longitudinal axial a subaxial (correspondiente a la Fig. 3C) x3,7. b. Ejemplar IMGPGö 495-104. Vista general de un fragmento de colonia no ramificada, x2. d. Ejemplar IMGPGö 495-101, paralectotipo. Morfología de los cálices. Obsérvese el desarrollo local de una cresta media, x10. e. Ejemplar IMGPGö 495-103. Vista general de un fragmento de colonia mostrando tres ramificaciones (flechas) a ambos lados de la rama principal (flecha doble). Obsérvese la presencia de una cubierta epitecal en la base de la ramificación izquierda, x2. g. Ejemplar IMGPGö 495-107. Sección transversal, zona axial. Obsérvese la pigmentación axial de las espinas murales y la ligera deformación de la tábula en su contacto, x22. h-j. Ejemplar IMGPGö 495-98, fotografías de tres láminas delgadas figuradas por Thomas (1905, p. 268). h. Sección transversal (ver también Figura 2), x3. i. Sección longitudinal (ver también Figura 5), x3. j. Sección tangencial (ver también Figura 2B), x4. Las fotografías a, f, h, i, han sido realizadas por R. Birenheide, el resto por Y. Plusquellec.

Cerro del Fuerte, Lochkovian. Lectotype and topotypes. a, c, f. Sample IMGPGö 495-100 figured by Thomas (1905, Plate. XII, fig. 20a). Lectotype. a. Overview of the branch previous to being sectioned in order to produce thin sections, showing a distal forking (triangles), x2. c. calices morphology, x5. f. axial to subaxial longitudinal section (see also Fig. 3C), x3,7. b. Sample IMGPGö 495-104. Overview of an unbranched colony fragment, x2. d. Sample IMGPGö 495-101, paralectotype. Calices morphology. Note sporadic development of a central crest, x10. e. Sample IMGPGö 495-103. Overview of a colony fragment presenting three branches (arrows) on both sides of the main stem (double arrow). Observe the development of an epithecal covering at the base of the left-hand forking, x2. $g$. Sample IMGPGö 495-107. Cross section, axial zone. Note the axial pigmentation of the wall spines and the slight tabulae deformation where they come into contact, x22. h-j. Sample IMGPGö 495-98. Photographs of three thin sections figured by Thomas (1905, p.268). h. Cross section (see also Fig. 2A), x3. i. Longitudinal section (see also Fig. 5 ), x3. $j$. Tangential section (see also Fig. 2B), x4. Photographs $a, f, h$, and $i$, were taken by $R$. Birenheide, and others by $Y$. Plusquellec. 

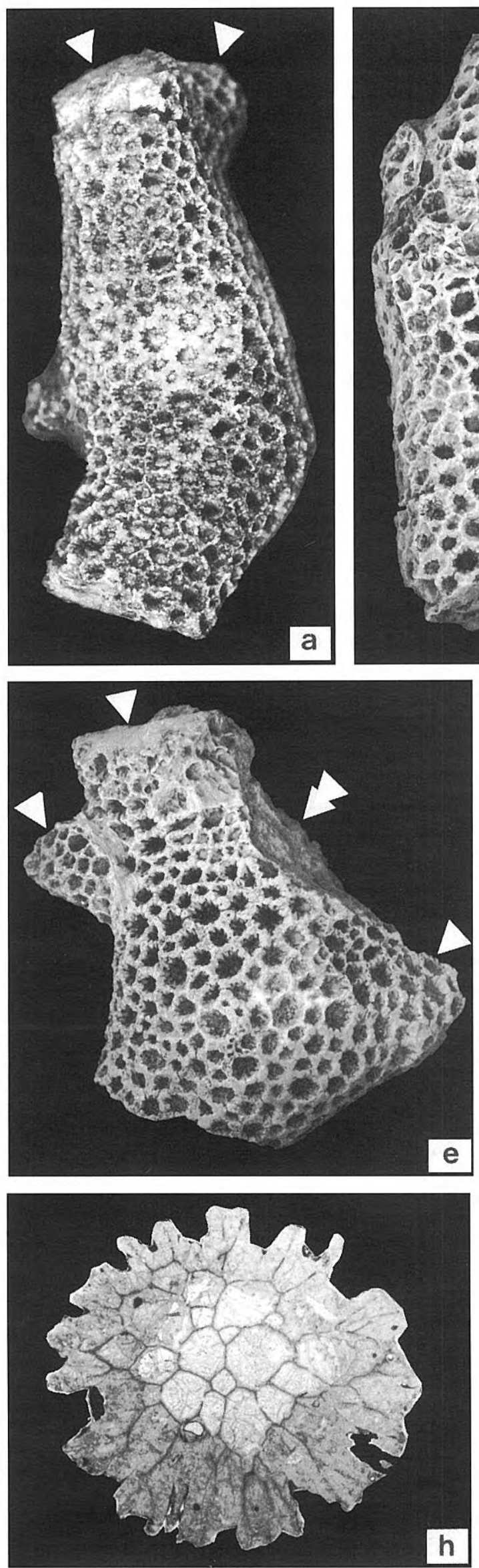
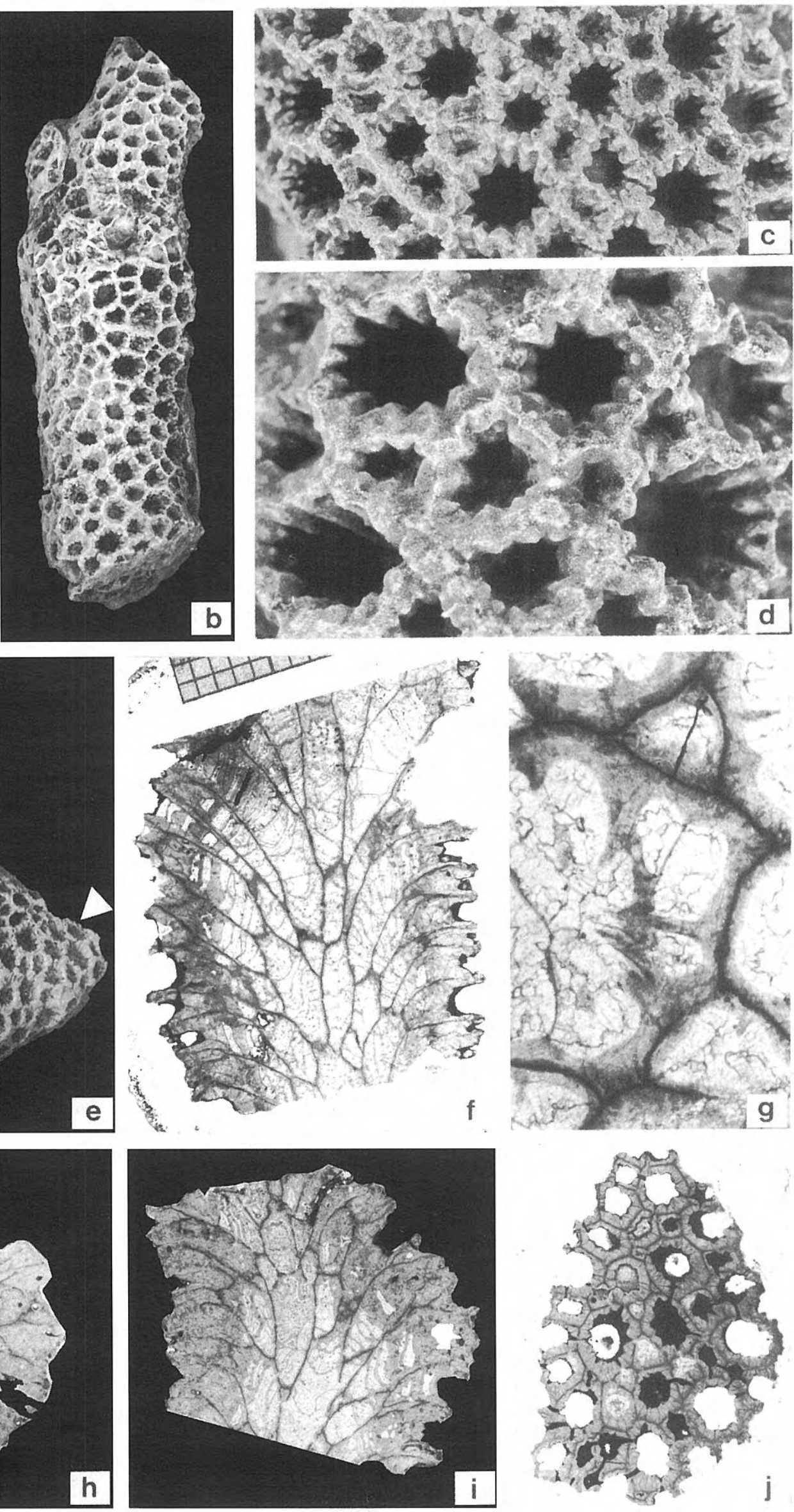
inclinadas, y que tienen sus raíces en la capa de tejido microlamelar que cubre la base de algunas tábulas.

\section{Microestructura}

En este apartado se describirá sucesivamente el aspecto de la microestructura del esqueleto en láminas de espesor petrográfico $(30 \mu \mathrm{m})$ y en láminas LFP (técnica de Lafuste, 1970).

Observaciones en láminas delgadas de tipo petrográfico

La pared de las coralitas en la zona axial aparece constituida por tres elementos.

1) Una lámina media oscura, estrecha, no sinuosa $y$ fundamentalmente continua. Las frecuentes interrupciones que se observan, tanto en sección transversal como en longitudinal, son debidas a la proximidad de poros murales (es decir, que se ha seccionado la pared ligeramente por encima, por debajo o lateralmente a un poro).

2) A ambos lados de la lámina media, o a veces en uno solo de sus lados, se desarrolla una capa relativamente gruesa de aspecto fibroso, con las "fibras" insertadas perpendicularmente a la lámina media (Fig. 2A). El espesor de la capa de "fibras" varía entre 20 y $80-90 \mu \mathrm{m}$ pero suele estar próximo a $50-70 \mu \mathrm{m}$.

3) Una capa clara, de grosor variable, constituida por elementos aplanados dispuestos paralelamente a la lámina media. Esta capa recubre la zona "fibrosa" o, más raramente, aparece en contacto directo con la lámina media oscura. Este esclerénquima, principalmente microlamelar (ver apartado siguiente) puede ser localmente muy delgado (de tal forma que tan sólo una buena lámina delgada y una observación atenta permiten descubrir su presencia) y, en este caso, la mayor parte de la pared está constituida por la doble zona fibrosa.

Las tábulas están desprovistas de capa "fibrosa" de tal forma que, en general, se encuentran reducidas a una delgada línea oscura y, cuando aparecen engrosadas, esta línea subyace a una capa de esclerénquima claro.

En la zona periférica se encuentran los mismos elementos que en la zona axial pero con algunas modificaciones:

1) La lámina media muestra una tendencia a la discontinuidad y a ser localmente reemplazada, particularmente en la zona calicinal, por estructuras claras de morfología más o menos losángica. Estas estructuras corresponden a ramilletes de fibras sucesivas (ver apartado siguiente) y parecen marcar la fase terminal del crecimiento del esqueleto.

2) La capa "fibrosa" se reduce o desaparece y, así, el esclerénquima microlamelar puede estar directamente en contacto con la lámina media. No se ha encontrado esta capa en la zona donde aparecen estructuras losángicas.

3) El esclerénquima microlamelar (o microlamelarlamelar, ver apartado siguiente) se engrosa considerablemente hasta formar las arrugas septales, en cuyo plano medio se desarrolla una fila de espinas (Fig. 2B).

Observaciones en láminas ultrafinas (LFP)

Los elementos fundamentales que conforman la pared y las tábulas son idénticos en la zona axial y en la periférica, por lo que su morfología será descrita globalmente (Fig. $6 \mathrm{~A})$.

Gránulos: La lámina media está constituida por gránulos isodiamétricos de aproximadamente $5 \mu \mathrm{m}$ de diámetro que forman una delgada capa de 10-15 $\mu \mathrm{m}$.

Fibroides: Según la zona considerada, la lámina media está recubierta o no por una capa formada por elementos alargados, dispuestos perpendicularmente a ella. Estos elementos presentan un contorno irregular y no parecen estar bien conservados. Tienen un diámetro de 6-7 $\mu \mathrm{m}$ y una longitud máxima observada de $35 \mu \mathrm{m}$. Estos cristales pueden ser asimilados a los fibroides descritos, por ejemplo, en Saouraepora (Plusquellec et al., 1993) ya que su ubicación es idéntica y su tamaño y morfología son muy similares.

Microlamelas y lamelas: El esclerénquima claro que constituye el espesor principal de la pared está formado por dos tipos de elementos aplanados y globalmente dispuestos de forma paralela a la lámina media, microlamelas y lamelas.

Las microlamelas (Fig. 6B) son relativamente gruesas, muy arqueadas, con su cara cóncava dirigida hacia el lumen y muestran una cupulación muy neta. Indican siempre, de forma clara, el sentido de acreción del esqueleto. Para un total de 20 medidas realizadas, la longitud (diámetro) oscila entre 13 y $33 \mu \mathrm{m}$; un $75 \%$ de estas medidas están comprendidas entre 18 y $29 \mu \mathrm{m}$, siendo la longitud más usual $24 \mu \mathrm{m}$. Por lo que respecta a la altura, el $95 \%$ de las medidas realizadas están comprendidas entre 8 y $13 \mu \mathrm{m}$. El $75 \%$ de los espesores medidos se sitúa entre 6 y $9 \mu \mathrm{m}$. Por comparación con las microlamelas tipo de Parastriatopora rhizoides, cuyo diámetro máximo es aproximadamente de 15-20 $\mu \mathrm{m}$ (Fernández-Martínez et al., 1999a), las microlamelas de Argentinella nov. gen. pueden entrar en la categoría de "grandes microlamelas" (Plusquellec y Tourneur, 1998).

La morfología de las lamelas es totalmente diferente a la de las microlamelas. Se trata de elementos planos, algunas veces ligeramente ondulados, más bien delgados y sin espesamiento notable en la zona media. Además, la cupulación está poco marcada por lo que la diferencia entre la forma de las caras proximal y distal se encuentra poco acentuada. Su longitud está comprendida generalmente entre 30 y $40 \mu \mathrm{m}$ para un espesor próximo a las $5 \mu \mathrm{m}$.

La coexistencia de microlamelas y lamelas en el seno del esclerénquima de Argentinella es indiscutible pero también existen elementos de morfología intermedia. Se trata de cristales de gran tamaño $(40$ a $50 \mu \mathrm{m})$, más gruesos que las lamelas típicas $(8-9 \mu \mathrm{m})$, de contorno más irregular y algunas veces ligeramente arqueados. En definitiva, parecen más próximos a las grandes microlamelas que a las lamelas clásicas.

Observación: En un trabajo inédito realizado por el fallecido J. Lafuste durante la década de los 70 , se han encontrado varias observaciones sobre la microestructura del ejemplar escogido como lectotipo para Argentinella nov. gen.; J. Lafuste había realizado en él tres láminas LFP (ligeramente gruesas), reconociendo la dualidad de los biocristales aplanados y dibujando una zona con microlamelas y otra con lamelas. Las formas y dimensiones de dichos elementos se corresponden perfectamente con las observaciones realizadas por nosotros en láminas de mejor calidad. 
En la pared, la disposición de las microlamelas y lamelas no parece estar regulada de forma rigurosa como ocurre, por ejemplo, en Syringopora (Lafuste et al., 1992b) donde se observa el paso de microlamelas a lamelas en el esclerénquima de la pared. Globalmente desde la lámina media (o desde la lámina media y su capa de fibroides) se observa la sucesión de microlamelas-lamelas (Fig. 6A) pero es frecuente encontrar lamelas típicas entre microlamelas bien caracterizadas. Además, en los espesamientos periféricos donde, en ocasiones, dominan las lamelas, pueden encontrarse paquetes de microlamelas.

En las proximidades de la lámina media, las microlamelas y lamelas se disponen paralelamente a un plano medio de la pared. Por otro lado, y a diferencia de lo que ocurre en los esclerénquimas lamelares típicos (Lafuste y Plusquellec, 1976), las lamelas no constituyen nunca conjuntos que se reencuentran bajo los ángulos bien marcados. El conjunto del escleréquima presenta, por tanto, una organización espacial que se traduce en afinidades microlamelares dominantes.

En el contacto de las espinas con la muralla, las microlamelas y lamelas se verticalizan curvándose en dirección distal, algo que se aprecia en muchos taxones con estos elementos microestructurales.

En conclusión, y a excepción de la zona de fibroides que puede ser considerada como una capa de transición -sin significado sistemático a nivel familiar- entre la lámina media y el esclerénquima mural, la pared de Argentinella nov. gen. puede ser interpretada como básicamente microlamelar (constituida por grandes microlamelas). Sin embargo, tanto la intercalación de lamelas entre microlamelas y viceversa, como la presencia de elementos de morfología intermedia, obliga a definir el esclerénquima mural como microlamelar-lamelar. Los espesamientos de las tábulas son de la misma naturaleza.

Trabéculas: En la pared, las estructuras constituidas por fibras divergentes se desarrollan en el emplazamiento de la lámina media y también en las espinas, tanto en las generadas en el esclerénquima mural como en las que aparecen sobre las tábulas.

En la parte media de la pared, los ramilletes de fibras están, generalmente, recristalizados en forma de 2 o 3 grandes monocristales ligeramente divergentes, causantes de la forma losángica y el aspecto hialino de estas estructuras. Las espinas han sufrido la misma transformación y solo raramente se puede observar un sistema de cristales aciculares de contorno sinuoso (longitud $20 \mu \mathrm{m}$, diámetro 2$2,5 \mu \mathrm{m})$ y débilmente divergentes.

La mala calidad de la conservación impide hacer la distinción propuesta por Cuif y Gautret (1991) para las diferentes unidades estructurales fibrosas: trabéculas s.s., "en chorro de agua" y "pennicilée" (aunque este último tipo está claramente ausente en el material estudiado). La recristalización parcial o total de las trabéculas en esqueletos donde las lamelas y microlamelas se encuentran bien conservadas es un fenómeno frecuente en los Tabulados (ver Lafuste y Plusquellec, 1987 para una figuración y descripción de este fenómeno en el género Ohiopora).

Por último, debemos indicar que, a nuestro conocimiento, esta es la primera vez que se describen, no la coexistencia de microlamelas y lamelas en un mismo taxón, sino las intercalaciones de lamelas entre las microlamelas en diferentes niveles del esclerénquima mural. Este hecho nos obliga a proponer una modificación en la diagnosis de la familia Parastriatoporidae.

\section{Discusión}

Puesto que se trata de un género monotaxónico, no hay posibilidades de discutir el estatus de esta especie. No obstante, quisiéramos indicar que el primer autor que re-estudió el material tipo aquí descrito, J. Lafuste, señaló a uno de los autores (F.T.) la presencia del género en el Devónico Inferior de Marruecos. Sin embargo, y hasta la fecha, no se ha encontrado en las colecciones norteafricanas ningún material que pueda atribuirse a Argentinella nov. gen.

\section{AGRADECIMIENTOS}

Los autores de este trabajo deseamos expresar nuestro sincero agradecimiento a Hans Jahnke (Institut und Museum für Geologie und Paläontologie, Göttingen) por el préstamo del material original estudiado y a Rudolf Birenheide por las fotografías del material tipo. Gracias, muy especiales, a Zarela Herrera (Universidad de Zaragoza) por su inestimable ayuda en la confección de los apartados relativos a localización del material, así como por su apoyo incondicional y rápida respuesta ante todo lo solicitado. Este trabajo se enmarca dentro de los Proyectos PB98-1563 y PICG 421 .

\section{BIBLIOGRAFÍA}

Baldis, B. A. 1971. La posición estratigráfica de Favosites argentina Thomas. Ameghiniana, 8, 77-82.

Baldis, B. A. 1975. El Devónico Inferior en la Precordillera Central. Parte I: Estratigrafía. Revista de la Asociación Geológica Argentina, 30, 53-83.

Benedetto, J. L., Racheboeuf, P. R., Herrera, Z., Brussa, E. D., et Toro, B. A. 1992. Brachiopodes et biostratigraphie de la Formation de Los Espejos, Siluro-Dévonien de la Précordillère argentine. Geobios, 25, 599-637.

Bodenbender, W. 1896. Beobachtungen uber Devon- und Gondwana-Schichten in der Argentinischen Republik. Zeitschrift geologie Gesellschaft, 743-772.

Bodenbender, W. und Kayser, E. 1896. Ueber Silur, Devon, Carbon und die Glossopteris-Stufe in der Gegend von Jachal im nordwestlichen Argentinien. Zeitschrift geologie Gesellschaft, 183-186.

Castellaro, H. A. 1966. Guía Paleontológica Argentina. Consejo Nacional de Investigaciones Científicas y Técnicas, Sección III (Faunas Silúricas) y IV (Faunas Devónicas). 164 pág.

Contreras, V. H. y Peralta, S. H. 1994. Revisión de la posición genérica de Favosites argentina Thomas (Cnidaria Tabulata), Devónico Inferior de la Precordillera de San Juan (Argentina). Resumen del VI Congreso Argentino de Paleontología y Bioestratigrafía, 
Trelew-Chubut, 1994, 54-55.

Cuif. J. P.and Gautret, P. 1991. Microstructural features of fibrous tissues in the skeletons of some chaetetid sponges. Courier Forschunginstitut Senckenberg, 164, 309-315.

Fernández-Martínez, E., Plusquellec, Y., Tourneur, F. y Herrera, Z. 1999a. Nueva especie de Tabulado del Devónico Inferior de Argentina. Revista Española de Paleontología, 14, 37-57.

Fernández-Martínez, E., Plusquellec, Y., Mistiaen, B. y Tourneur, F. 1999b. Crenulipora Le Maître, 1956 (Tabulata, Devónico): Revisión del material original y estado actual de conocimientos. Temas GeológicoMineros ITGE, 26, 594-598.

Herrera, Z. A. 1993. Nuevas precisiones sobre la edad de la Formación Talacasto (Precordillera Argentina) en base a su fauna de braquiópodos. XII Congreso Geológico Argentino y II Congreso de Exploración de Hidrocarburos (Mendoza, 1993). Geología y Recursos Naturales de Mendoza, Actas, 2 (Ed. V. A. Ramos), 289295.

Herrera, Z. A. 1995. The Lower Devonian chonetoidean brachiopods from the Argentine Precordillera. Documents des Laboratoires de Géologie de Lyon, 136, 101-147.

Lafuste, J. 1970. Lames ultra-minces à faces polies. Procédé et application à la microstructure des Madréporaires fossiles. Comptes rendus de l'Académie des Sciences, Paris, 270, 679-681.

Lafuste, J. et Plusquellec, Y. 1976. Kerforneidictyum n. gen. (Tabulata, Dévonien). Morfologie et microstructure. Bulletin de la Société géologique de France, 18, 16991711.

Lafuste, J. et Plusquellec, Y. 1987. Structure et microstructure de Favosites cylindrica Michelin 1847, espèce-type de Ohiopora n. gen. (Tabulata, Dévonien). Canadian Journal of Earth Sciences, 24, 1465-1477.

Lafuste, J., Fernández-Martínez, E. y Tourneur, F. 1992a.
Parastriatopora (Tabulata) de las Calizas del Lorito (Devónico Inferior, Provincia de Córdoba): Morfología y microestructura. Revista Española de Paleontología, 7, 3-12.

Lafuste, J., Semenoff-Tian-Chansky, P. et Tourneur, F. 1992b. Succession microlamelles-lamelles dans le sclérenchyme pariétal de Syringopora Goldfuss, 1826 (Tabulata, Carbonifère). Bulletin du Muséum national d'Histoire naturelle, Paris, $4^{\text {ème }}$ sér., 14, C(3-4), 249265.

Le Hérissé, A., Rubinstein, C. and Steemans, P. 1997. Lower Devonian Palynomorphs from the Talacasto Formation, Cerro del Fuerte Section, San Juan Precordillera, Argentina. Acta Universitatis Carolinae Geológica, 40, 497-515.

Oliver, W. A. Jr. 1966. Description of dimorphism in Striatopora flexuosa Hall. Palaeontology, 9, 448-454.

Plusquellec, Y. et Tourneur, F. 1998. Persistance de Favositides microlamellaires (Cnidaria, Tabulata) dans le Dévonien. Comptes rendus Académie des Sciences, Paris, Sciences de la terre et des planètes, 326, 283-289.

Plusquellec, Y., Tourneur, F. et Lafuste, J. 1993. Saouraepora nouveau genre de Micheliniidae (Tabulata) du Dévonien du Nord Gondwana et du Carbonifère d'Amérique du Nord. Palaeontographica A, 227, 1-86.

Thomas, I. 1905. Neue Beitrage zur Kenntnis der devonischen Fauna Argentiniens. Zeitschrift der Deutschen geologischen Gesellschaft, 57(1), 233-290.

Tourneur, F. 1992. Présence du genre Parastriatopora Sokolov 1949 (Tabulata) dans le Dévonien supérieur de la Bolivie: implications paléogéographiques. Table ronde européenne «Paléontologie et Stratigraphie en Amerique Latine», Lyon, 7-9 juillet, 1992, résumés, p. 47.

Tourneur, F. , Plusquellec, Y., Fernández-Martínez, E. M. and Díaz Martínez, E. 2000. Revision of Parastriatopora gigantea (Knod, 1908) (Anthozoa, Tabulata) from the Devonian of Bolivia. Geobios, 33, 709-724. 